- But the signal for that event was not very strong, which means that it could be a fluke. "I think people should get excited about it, but they should also be aware that the significance is much lower" than in many previous events, he says. LIGO and Virgo have already detected gravitational waves - faint ripples in the fabric of space-time - from two types of cataclysmic event: the mergers of two black holes, and the collision of two neutron stars. The latter are small, ultra-dense objects formed after the collapse of stars more massive than the Sun.

The latest event, provisionally labelled S190426c, seems to have occurred around 375 megaparsecs (1.2 billion light years) away, the LIGO-Virgo team calculated. For both of last week's detections, the researchers also drew 'sky maps', showing where the gravitational waves are most likely to have originated, and sent this information out as public alerts, so that astronomers around the world could begin searching the sky for light from the events. Matching gravitational waves to other forms of radiation in this way can produce much more information about the event than can either form alone.

For both detections, astronomers worldwide raced to make follow-up observations in different wavelengths - a practice known as multimessenger astronomy — but have been unsuccessful so far, perhaps because the events were far away and the signal was too faint.

For the 26 April event, part of the sky map extended over the north polar region, where telescope coverage is poor, making the search more difficult, says Daniel Perley, an astronomer at Liverpool John Moores University, UK, who was involved in the follow-up searches. Another possibility is that the source was hidden behind the Milky Way's 'dustier' regions. As days went by, the odds of finding the source became longer. "The expectations are not too high at this point," Perley says.

If the 26 April event was not a black holeneutron star merger, it was probably also a collision of neutron stars, which would bring the total detections of this type up to three.

But seeing a black hole sweep up a neutron star could help to answer a host of questions. These long-sought systems are thought to originate from binary stars of very different masses. The orbits the two objects trace in the final phases of their approach could be rather different from those followed by pairs of black holes. In the black hole-neutron star case, the more-massive black hole would twist space around it as it spins. "The neutron star will be swirled around in a spherical orbit rather than a quasi-circular orbit," says B. S. Sathyaprakash, a LIGO theoretical physicist at Pennsylvania State University. This gravitational complexity means "neutron star-black hole systems can be more powerful test beds for general relativity".

Moreover, the gravitational waves and the companion observations from astronomers could reveal what happens in the final phases before such a merger. As tidal forces tear the neutron star apart, they could help astrophysicists to solve a long-standing mystery: what state is matter in inside these ultra-compact objects?

The LIGO-Virgo collaboration began its current observing run on 1 April, and researchers had expected to see roughly one merger of black holes per week and one of neutron stars per month. So far, those predictions have been met - the observatories have also seen several black-hole mergers this month. "This is just amazing," says Mansi Kasliwal, an astrophysicist at the California Institute of Technology in Pasadena who works on follow-up searches. "The Universe is fantastic." -

\title{
Biggest Denisovan fossil yet spills ancient human's secrets
}

\section{Jawbone identified using only protein analysis reveals that the species was widespread.}

\section{BY MATTHEW WARREN}

$\mathrm{S}$ cientists have uncovered the most complete remains yet from the mysterious ancienthominin group known as the Denisovans. The jawbone, discovered high on the Tibetan Plateau and dated to more than 160,000 years ago, is also the first Denisovan specimen found outside the Siberian cave in which the species was discovered a decade ago - confirming suspicions that the hominin was more widespread than the fossil record currently suggests.

The research marks the first time an ancient human has been identified solely through protein analysis. In the absence of usable DNA, scientists examined proteins in the specimen's teeth, raising hope that more fossils could be identified even when DNA is not preserved.

"This is fantastic work," says Katerina Douka, an archaeologist at the Max Planck Institute for the Science of Human History in Jena, Germany, who was not involved in the research. "It tells us that we are looking at the right area".

So far, everything scientists have learnt about Denisovans has come from a handful of teeth and bone fragments from Denisova Cave in Russia’s Altai Mountains. But many expected that it was only a matter of time before traces of the group were found elsewhere. Some modern humans in Asia and Oceania carry traces of Denisovan DNA, raising the possibility that the

hominin lived far away from Siberia. And some researchers thought that unclassified hominin fossils from China could be Denisovan.

The latest fossil, described in Nature ${ }^{1}$, consists of half of a lower jaw, with two complete teeth. It was found by a monk in Baishiya Karst

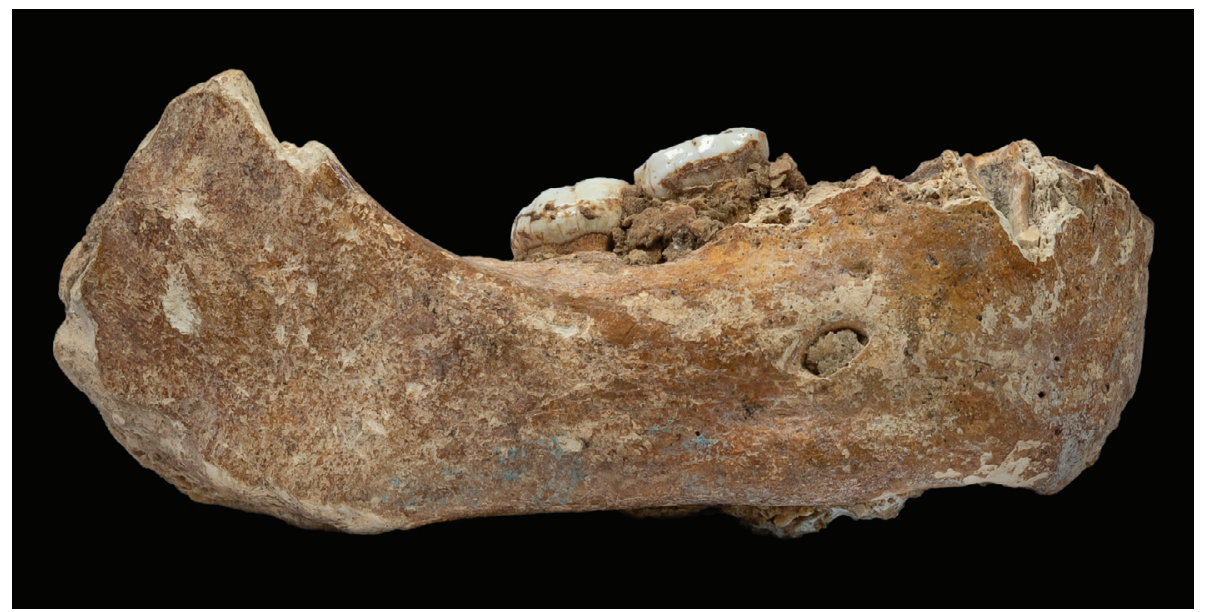

A Denisovan jawbone was discovered on the Tibetan Plateau at an altitude of more than 3,000 metres. 
Cave in China in 1980, and passed on to Lanzhou University. But it wasn't until the 2010s that archaeologist Dongju Zhang and her colleagues began studying the bone.

The team faced a problem. The remains at Denisova Cave had all been identified because they still contained some DNA, which could be compared with genetic sequences from other ancient humans. But there was no DNA left in the jawbone.

Instead, the scientists looked for ancient proteins, which tend to last longer than DNA. In dentine from the teeth, they found collagen proteins suitable for study. The team compared these with equivalent proteins in great apes including Denisovans and Neanderthals, and found that they lined up closest with samples from Denisovans.

Previous work ${ }^{2}$ identified Neanderthal remains using both proteins and DNA — but the success of the latest study could lead to a greater emphasis on getting ancient proteins out of fossils that haven't yielded DNA, says Chris Stringer, a palaeoanthropologist at the Natural History Museum in London. The method could prove particularly useful for older samples or those from warmer climates, where DNA degrades quickest.

\section{THE ROOF OF THE WORLD}

The altitude of the new Denisovan's home - 3,280 metres above sea level — surprised researchers, and helps to solve a mystery about Denisovans' genetic contribution to modern Tibetans. "It is astonishing that any ancient humans were at that altitude," says Stringer.

Some Tibetans have a variant of a gene called EPAS1 that reduces the amount of the oxygen-carrying protein haemoglobin in their blood, enabling them to live at high altitudes with low oxygen levels. Research$\mathrm{ers}^{3}$ had thought that this adaptation came from Denisovans, but this was difficult to reconcile with Denisova Cave's relatively low altitude of 700 metres. The latest study suggests that Denisovans evolved the adaptation on the Tibetan Plateau and passed it to Homo sapiens when the species arrived around 30,000-40,000 years ago, says coauthor Frido Welker, a molecular anthropologist at the University of Copenhagen.

If Denisovans in Asia were adapted to high altitudes, similar sites could harbour more of their remains. And the jawbone is likely to prompt scientists to reconsider classification of other ancient-human remains. "We can kind of work ourselves through the fossil record, and link up more and more specimens with the Denisovans," says Bence Viola, a palaeoanthropologist at the University of Toronto in Canada. .

1. Chen, F. et al. Nature https://doi.org/10.1038/ s41586-019-1139-x (2019)

2. Welker, F. et al. Proc. Natl Acad. Sci. USA 113

11162-11167 (2016).

3. Huerta-Sánchez, E. et al. Nature 512, 194-197 (2014).

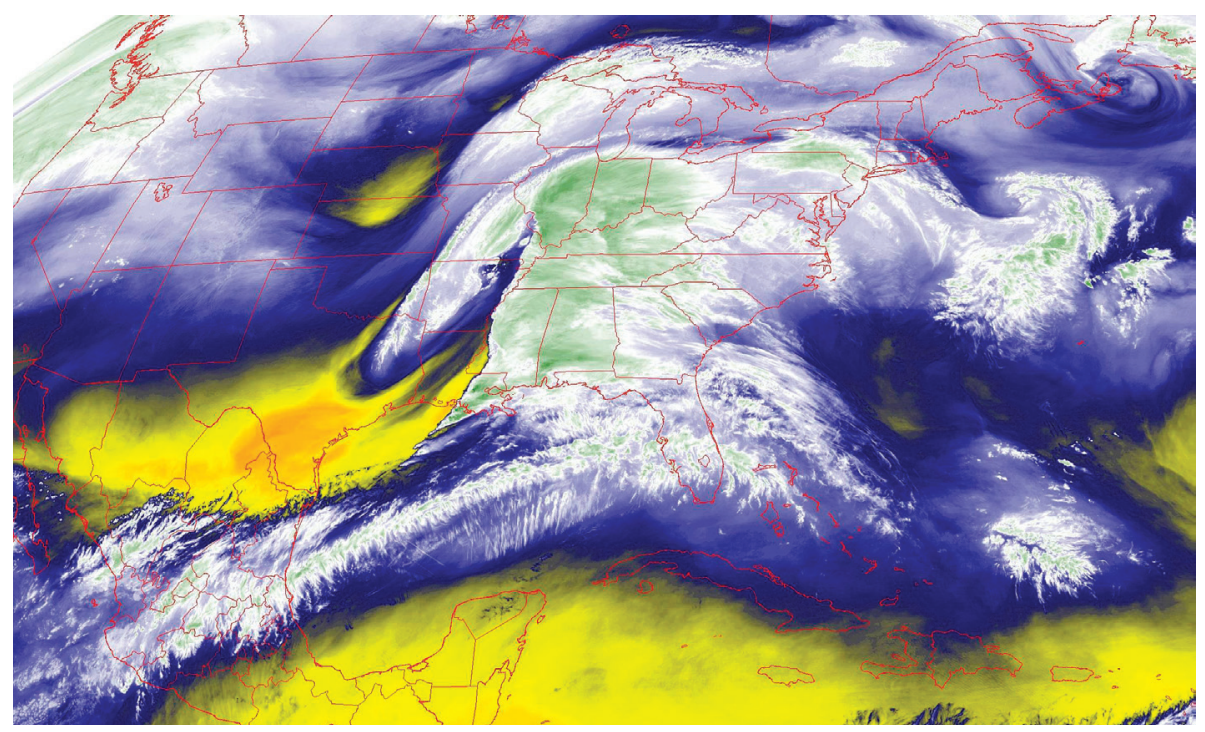

Satellite images of water-vapour levels are used to predict weather patterns.

ATMOSPHERIC SCIENCE

\section{G data networks threaten forecasts}

\section{Wireless technology could interfere with Earth observations.}

\section{BY ALEXANDRA WITZE}

? he US government has begun auctioning off blocks of wireless radio frequencies to be used for the nextgeneration cellular communications network, known as $5 \mathrm{G}$. But some of these frequencies lie close to those that satellites use for crucial Earth observations - and meteorologists are worried that $5 \mathrm{G}$ mobile-phone transmissions could hamper their data collection.

Unless regulators or telecommunications companies take steps to reduce the risk of interference, Earth-observing satellites flying over areas of the United States with $5 \mathrm{G}$ wireless coverage won't be able to detect water vapour in the atmosphere accurately. Meteorologists rely on that information, and without it, weather forecasts worldwide are likely to suffer.

"This is a global problem," says Jordan Gerth, a meteorologist at the University of Wisconsin-Madison.

The US National Oceanic and Atmospheric Administration (NOAA) and NASA are currently locked in a high-stakes negotiation with the Federal Communications Commission (FCC), which oversees US wireless networks. NOAA and NASA have asked the FCC to help them to protect frequencies used for Earth observations from interference as $5 \mathrm{G}$ rolls out. But the FCC auctioned off the first chunk of the $5 \mathrm{G}$ spectrum with minimal protection. The sale ended on 17 April, reaping nearly US $\$ 2$ billion.

Because the United States is such a large communications market, the government's decisions about how to deploy $5 \mathrm{G}$ are likely to influence global discussions on the technology. Regulators from around the world will meet in October in Egypt to set international agreements for which frequencies companies can use for $5 \mathrm{G}$ transmissions, and what level of interference with Earth observations is acceptable.

Astronomers, meteorologists and other scientists have long worked to share the spectrum with other users, sometimes shifting to different frequencies. But "this is the first time we've seen a threat to what I'd call the crown jewels of our frequencies", says Stephen English, a meteorologist at the European Centre for Medium-Range Weather Forecasts in Reading, UK.

They include the 23.8-gigahertz frequency, at which water vapour in the atmosphere emits a faint signal. Satellites monitor energy radiating from Earth at this frequency to assess humidity in the atmosphere below. Forecasters feed these data into models to predict how storms and other weather systems will develop.

But a $5 \mathrm{G}$ station transmitting at nearly the same frequency will produce a signal that looks like that of water vapour. "We wouldn't know that that signal is not completely natural," says Gerth. Forecasts would be less accurate if scientists used those bad data. 